Pacific Journal of Mathematic 


\title{
AUTOMORPHISM GROUPS OF OPERATOR ALGEBRAS
}

\author{
RICHARD H. HERMAN
}

The general setting of this paper is that of a von Neumann algebra, $M$, with weight, $\varphi$, and a group, $G$, of automorphisms which commute with the modular automorphism group associated with this weight.

The first section is devoted to the question of when the given weight is invariant under the action of $G$. Should $G$ leave the center of $M$ elementwise fixed results have been obtained by Pedersen, St $\varnothing$ rmer, Takesaki, and the present author. If, alternatively, it is assumed that $\varphi$ is invariant under a subgroup, $H$, of $G$, then by requiring an ergodic action of $H$ on the center of $M$ it is shown here that $\varphi$ must be (semi-) invariant under the action of $G$. This is done with the aid of some technical assumptions $H$. Less demanding hypothesis are shown to lead to a $G$-invariant weight bicommuting with the given weight.

Section two is mostly devoted to a discussion of ergodicity in time or the requirement that the centralizer of a given state coincide with the center of the von Neumann algebra in question. In particular, we show that if the fixed point algebra of a given group of automorphisms, commuting with the modular automorphism group of the given state, is semifinite, then it is contained in the center if we have ergodicity in time. We also show, in the spirit of [6], that if the centralizer is a factor, then there is no semifinite von Neumann algebra which properly contains the centralizer and is invariant under the modular automorphism group.

I. The general setting for this section will be that of a von Neumann algebra, $M$, acted upon by two commuting automorphism groups, $\left\{\alpha_{g}: g \in G\right\}$ and $\left\{\sigma_{t}^{\varphi}: t \in R\right\}$. The latter will be the unique modular group associated with a normal, faithful semifinite, weight $\varphi$, and the former will always be assumed continuous in that $g \rightarrow \psi\left(\alpha_{g}(x)\right)$ is continuous on $G$ for arbitrary $\psi \in M *, x \in M$.

Recall that a weight, $\varphi$, on a von Neumann algebra $M$, is a mapping from $M^{+}$to $[0, \infty]$ satisfying

(i ) $\varphi(\alpha x)=\alpha \varphi(x) ; \alpha \in R^{+}, x \in M^{+}$

(ii) $\varphi(x+y)=\varphi(x)+\varphi(y) ; x, y \in M^{+}$.

The weight is said to be normal if in addition there is a set of bounded normal positive functionals on $M$ such that

(iii) $\varphi(x)=\sup \omega_{i}(x)$ for each $x \in M^{+}$.

Further, the weight is semifinite if the linear span $m$ of the set 


$$
\mathfrak{m}^{+}=\{x \in M+\mid \varphi(x)<\infty\}
$$

is $\sigma$-weakly dense in $M$. Faithfulness requires that $\varphi(x)=0$ implies $x=0$ for all $x \in M^{+}$.

It turns out the most important additional stipulation on the group of automorphisms $\left\{\alpha_{g}\right\}$ is its action on the center, $\mathscr{r}$, of $M$. The case where the center is pointwise fixed has been discussed in [5] and [12]. The action of $\left\{\alpha_{g}\right\}$ is sain to be ergodic on $\mathscr{z}$ if there are no elements in $\mathscr{F}$ fixed by all $\alpha_{g}, g \in G$, save the scalars.

TheOREm 1. Let $\varphi, \sigma_{t}^{\varphi}$ and $\alpha_{g}$ be as above. Suppose that for a normal subgroup $H$ of $G$ we have $\varphi \circ \alpha_{h}=\varnothing$ for $h \in H$. If $\left\{\alpha_{h}: h \in H\right\}$ acts ergodically on $\mathscr{F}$ then $\varphi\left(\alpha_{g}(x)\right)=\lambda_{g} \varphi(x)$ for $x \in M$ where $\lambda_{g}$ is a continuous homomorphism of $G \rightarrow R^{+}$.

Proof. Since $\alpha_{g}$ commutes with $\sigma_{t}^{\varphi}$ it follows, [12], that

$$
\varphi\left(\alpha_{g}(x)\right)=\varphi\left(z_{g} x\right)
$$

where $z_{g}$ is a unique positive element affiliated with $\mathscr{z}$.

If $h \in H$ then

$$
\begin{aligned}
\varphi\left(\alpha_{g}\left(\alpha_{h}(x)\right)\right. & \left.=\varphi\left(\alpha_{g h}(x)\right)\right) \\
& =\varphi\left(\alpha_{h^{\prime} g}(x)\right) \text { for some } h^{\prime} \in H \text { by the normality of } H \\
& =\varphi\left(\alpha_{g}(x)\right) .
\end{aligned}
$$

Thus the weight $\phi \circ \alpha_{g}$ is invariant under $\left\{\alpha_{h}: h \in H\right\}$. Its RadonNikodym derivative, $z_{g}$, is then affiliated with those elements in $\mathscr{z}$ fixed by $\left\{\alpha_{h}: h \in H\right\}$. This is the scalars by assumption. Hence $\varphi\left(\alpha_{g}(x)\right)=\lambda_{g} \varphi(x)$ and the homomorphic nature of $g \rightarrow \lambda_{g}$ follows easily. The continuity is then a consequence of the lower semicontinuity of $g \rightarrow \lambda_{g}$ which follows from (iii).

We would like to avoid the normality restriction on $H$. This can be done if we restrict our attention to state and assume $H$ is "large" in $G$.

THEOREM 2. Let $G$ be a topological group with closed subgroup $H$ such that the homogeneous space of right cosets, $H \mid G$, is locally compact and supports a finite G-invariant, regular, Borel measure $\mu$. Let $\left\{\alpha_{g}\right\}$ and $\left\{\sigma_{t}^{\varphi}\right\}$ be commuting automorphisms of $M$. If $\varphi$ is a normal, faithful, state on $M$ and $\left\{\alpha_{h}: h \in H\right\}$ acts ergodically on $\mathcal{F}$ and leaves $\phi$ invariant, then $\varphi$ is invariant under the action of all of $\left\{\alpha_{g}: g \in G\right\}$.

Proof. Let $\dot{g}$ denote the coset $H_{g}$. Then we have 


$$
\varphi\left(\alpha_{h g}(x)\right)=\varphi\left(\alpha_{g}(x)\right) \text { and so }
$$

$\dot{g} \rightarrow \varphi\left(\alpha_{i j}(x)\right)\left(=\varphi\left(\alpha_{g}(x)\right)\right)$ defines a continuous function in $H \mid G$.

Let

$$
\psi(x)=\int_{H \backslash G} \varphi\left(\alpha_{g}(x)\right) d \mu(g) .
$$

Then $\psi$ is a normal [7], $G$-invariant, faithful state on $M$, which is easily seen to satisfy the KMS boundary condition with respect to $t \rightarrow \sigma_{t}^{\circ}$ ( $\psi$ is in the norm closed convex hull of $\left.\left\{\varphi \circ \alpha_{g}\right\}\right)$.

Thus $\psi(x)=\varphi(z x)$ where $z \geqq 0$ and $z \in \mathscr{z}$.

As in Theorem 1 we conclude that $z$ is a scalar $(=1)$ and since $\psi$ is $G$-invariant, so is $\varphi$.

Definition. A von Neumann algebra acted upon by a group of automorphism $\left\{\alpha_{g}: g \in G\right\}$ is said to be $G$-finite if for $x \in M^{+}, x \neq 0$, there exists a $G$-invariant state $\psi$ such that $\psi(x)>0$. In this case there exists a normal faithful projection, $\varepsilon_{G}$, of $M$ onto $M_{G}=\left\{x: \alpha_{g}(x)=\right.$ $x, g \in G\},[8]$.

In the above we have sought to obtain the $G$-invariance of a given weight, $\varphi$. Suppose instead that we are willing to settle for obtaining a $G$-invariant weight "closely related" to $\varphi$. Suppose then that $\varphi$ is a normal, faithful state on $M$ and $M$ is $G$-finite. If $\alpha_{g}$ commutes with $\sigma_{t}^{\circ}$ then we can easily see that $\varphi \circ \varepsilon_{G}$ is a faithful, normal state satisfying the KMS boundary condition with respect to $t \rightarrow \sigma_{t}^{\varphi}$. However, if $\varphi$ is only assumed to be a semifinite weight then one cannot necessarily conclude the semifiniteness of $\varphi \circ \varepsilon_{G}$.

With some additional restrictions and work of Størmer we can obtain a result in this direction.

First some definitions are needed.

Definition. A faithful normal semifinite weight $\varphi$ is said to be strictly semifinite if

(a) $M$ is $\left\{\sigma_{t}^{\varphi}: t \in R\right\}$-finite in the sense of the above definition equivalently (b). The restriction of $\varphi$ to $M_{\varphi}=\left\{x: \sigma_{t}^{\varphi}(x)=x, t \in R\right\}$ is a faithful, normal, semifinite trace. (See [1] for other equivalent statements.)

Definition. Two faithful, normal, semifinite weights, $\varphi$ and $\psi$ are said to bicommute if $\varphi(x)=\psi(z x)$ for all $x \in M$, where $z$ is positive, nonsingular operator affiliated with $\mathscr{\varkappa}_{\psi}=M_{\psi} \cap M_{\psi}^{\prime}$. (It is frequently the case that $\mathscr{2} \psi$ coincides with the relative commutant of $M_{\psi}$, [2].)

THEOREM 3. Let $\varphi$ be a strictly semifinite, normal, faithful weight on $M$ with modular automorphism group $t \rightarrow \sigma_{t}^{\varphi}$. Suppose 
that, $\left\{\alpha_{g}: g \in G\right\}$ is a group of automorphism of $M$ commuting with the modular automorphism group of $\varphi$ and that $M$ is a G-finite von Neumann algebra. If $G$ acts ergodically on the center of $M_{\varphi}\left(=\mathscr{Z}_{\varphi}\right)$ or leaves $\mathscr{Z}_{\varphi}$ elementwise invariant, then there exists a strictly semifinite, normal, faithful weight, $\psi$, bicommuting with $\varphi$ such that $\psi$ is G-invariant. If the action of $G$ is ergodic on $\mathcal{F}_{\varphi}$ then the weight \& is unique up to a positive multiple.

Proof. Since $\varphi$ is strictly semifinite, $M_{\varphi}$ is a semifinite von Neumann algebra. Further, the fact that $\alpha_{g}$ commutes with $\sigma_{t}^{\circ}$ means that $\alpha_{g}$ is an automorphism of $M_{\varphi}$, and thus that $M_{\varphi}$ is $G$-finite. The action of $\left\{\alpha_{g}: g \in G\right\}$ on $M_{\varphi}$ allows us to apply results of Størmer [13, $14,15]$ to obtain a $G$-invariant, faithful, normal, semifinite trace, $\tau$, on $M_{\varphi}$. But $\varphi$ is such a trace and so

$$
\tau(x)=\varphi(z x) \text { for all } x \in M_{\varphi} \text { where }
$$

$z \geqq 0$ and $z \eta \mathscr{Z C}_{\varphi}$.

Define

$$
\psi(x)=\tau \circ \varepsilon_{\varphi}(x) \text { for all } x \in M
$$

where $\varepsilon_{\varphi}$ is the normal, faithful projection of $M$ onto $M_{\varphi}$ the existence of which follows from the strict semifiniteness of $\varphi$, (see definitions above).

Recall, [8], that for $x \in M, \varepsilon_{\varphi}(x)$ is the unique point in $\bar{c} \bar{o}^{s t}\left\{\sigma_{t}^{\varphi}(x)\right\} \cap M_{\varphi}$.

Then

$$
\psi(x)=\tau\left(\varepsilon_{\varphi}(x)\right)=\varphi\left(z \varepsilon_{\varphi}(x)\right) .
$$

For $y \in M ; \varphi(z y)$ is defined to be $\lim _{\varepsilon \downarrow 0} \varphi\left(z_{\varepsilon} y\right)$ where $z_{\varepsilon}=z(1+\varepsilon z)^{-1}$. (See the remarks preceding Proposition 4.2 of [12].) With this comment we conclude that

$$
\psi(x)=\varphi(z x) .
$$

Now $\psi$ is $G$-invariant, since $\tau \circ \varepsilon_{\varphi}$ is. From [12] we have $\sigma_{t}^{\psi}(x)=$ $z^{i t} \sigma_{t}^{\varphi}(x) z^{-i t}$, so that $M_{\psi} \supseteqq M_{\varphi}$. We conclude that every $\sigma_{t}^{\varphi}$-invariant state is $\sigma_{t}^{\prime}$-invariant and thus that $\psi$ is strictly semifinite.

Suppose $\widetilde{\psi}$ is another such weight and $G$ acts ergodically in $\mathscr{L}_{\varphi}$. We claim $\widetilde{\psi}$ restricted to $M_{\varphi}$ is a (normal) semifinite, trace. Since $M_{\tilde{\psi}} \supseteqq M_{\varphi}, \widetilde{\psi}$ is clearly a normal trace in $M_{\varphi}$. But $\widetilde{\psi}(x)=\varphi\left(z_{1} x\right)$ where $z_{1}$ is a positive operator affiliated with the center of $M_{\varphi}$. Hence the argument of Proposition 4.2 of [12] shows that $\tilde{\psi}$ restricted to $M_{\varphi}$ is semifinite. Considering the restrictions of $\tilde{\psi}$ and $\psi$ to $M_{\varphi}$ we see, by the invariance of $\widetilde{\psi}$ and $\psi$, that the Radon-Nikodym derivative 
of $\tilde{\psi}$ with respect to $\psi$ is affiliated with the fixed points in the center of $M_{\varphi}$ and thus $\tilde{\psi}$ is a positive multiple of $\psi$. (This last argument is given by Størmer in [13].)

THeOREM 4. With the setting as in Theorem 3, a G-invariant weight, $\Psi$, bicommuting with $\varphi$ exists without assumptions concerning the action of $G$ on $M_{\varphi}$, if $M_{*}$ is separable.

Proof. Nest, [10], has shown that a semifinite, $G$-finite von Neumann algebra with separable predual admits a faithful, normal semifinite $G$-invariant trace.

CoRollary 5. With the same hypothesis as in Theorem 3, if $\mathscr{\varkappa}_{\varphi}=\{\lambda I\}$ (e.g., if $\phi$ is maximal [6]) then $\varphi$ is G-invariant.

The following is in the spirit of Theorems 1 and 2 and is immediate from Theorem 3 but we offer an alternative proof.

COROLlary 6. Suppose the setting to be the same as in Theorem 3 and in addtion suppose there exists a subgroup $H \cong G$ such that $\varphi\left(\alpha_{h}(x)\right)=\varphi(x), \quad h \in H, x \in M$. If $H$ acts ergodically on $\mathscr{Z}_{\varphi}$ then $\varphi\left(\alpha_{g}(x)\right)=\varphi(x)$ for all $g \in G, x \in M$.

Proof. Again we restrict our attention to $M_{\varphi}$ and obtain two semifinite, normal, faithful traces $\varphi$ and $\tau$ where $\tau$ is $G$-invariant. Let $\psi=\varphi+\tau$. Then $\psi$ is a semifinite, $H$-invariant, trace. There exists unique $z_{1}, z_{2} \in \mathscr{z}_{\varphi}$ such that

$$
\begin{aligned}
& \varphi(\cdot)=\psi\left(z_{1} \cdot\right) \\
& \tau(\cdot)=\psi\left(z_{2} \cdot\right) .
\end{aligned}
$$

But, $\varphi$ and $\tau$ are $H$-invariant. This puts $z_{1}, z_{2} \in M_{H} \cap \mathscr{Z}_{\varphi}=\{\lambda I\}$. The weight $\varphi$ is then a multiple of $\tau$ on $M_{\varphi}$ and hence is $G$-invariant there. But

$$
\begin{aligned}
\varphi\left(\alpha_{g}(x)\right)=\varphi\left(\varepsilon_{\varphi}\left(\alpha_{g}(x)\right)\right) & =\varphi\left(\alpha _ { g } \left(\varepsilon_{\varphi}(((x)))\right.\right. \\
& =\varphi\left(\varepsilon_{\varphi}(x)\right)=\varphi(x)
\end{aligned}
$$

II. In $\S I$ we considered the affect of an automorphism group which commuted with a given modular automorphism group and in some sense acted ergodically. Of course the center of $M$ is always elementwise fixed be any modular automorphism group and so we shall consider consequences of what is referred to as "ergodicity in time" namely the requirement that $M_{\varphi}=\mathscr{z}$. There seems to be no advantage to working with weights at this point and so we stick to states. 
We first prove a related result regarding the centralizer, $M_{\varphi}$, the proof of some being in the spirit of what follows (cf. [6]).

THEOREM 7. Suppose $\varphi$ is a normal, faithful state and $M_{\varphi} \subseteq$ $N \leqq M$ where $N$ is a von Neumann algebra invariant under $t \rightarrow \sigma_{t}^{\varphi}$. If $M_{\varphi}$ is a factor then

(i) $N$ is a factor.

(ii) If $N$ is semifinite then $N=M_{\varphi}$, i.e., there are no invariant semifinite von Neumann algebras properly containing $M_{\varphi}$.

Proof. Since $N$ is invariant, the center of $N$ is elementwise fixed by $t \rightarrow \sigma_{t}^{\circ}$. Thus $N \cap N^{\prime} \subseteq M_{\varphi} \cap M_{\varphi}^{\prime}=\{\lambda I\}$.

Suppose now that $N$ is semifinite. By the invariance of $N$, $\sigma_{t}^{\varphi}(x)=u_{t} x u_{-t}$ for $x \in N$, where the $u_{t}$ form a one parameter unitary group in $N$, [16]. Since $u_{s}, \mathrm{~s} \in R$ is fixed by $\sigma_{t}^{\varphi}, u_{s} \in M_{\varphi}$. But $N \supseteqq M_{\varphi}$, so $u_{s} \in \mathscr{z}_{\varphi}=\{\lambda I\}$ and thus $\sigma_{t}^{\varphi}(x)=x$ for all $x \in N$.

We remark here that if $M$ is in case $\mathrm{III}_{\lambda}, 0 \leqq \lambda<1$ this result is known [2], [6]. For under these circumstances, $M_{\varphi}$ is a factor if and only if $M_{\varphi}^{\prime} \cap M=\{\lambda I\}$, [2].

Now let $\left\{\alpha_{g}: g \in G\right\}$ be an automorphism group of $M$, commuting with the modular automorphism group $t \rightarrow \sigma_{t}^{\varphi}$, of a given faithful, normal state $\varphi$.

THEOREM 8. If in the setting just described $M_{\varphi}=\mathscr{Z}$ and $M_{G}$ is semifinite then $M_{G} \subseteq \mathscr{Z}$.

Proof. Again we have $\sigma_{t}^{\varphi}$ is inner on $M_{G}$ viz; there exist $t \rightarrow$ $v_{t} \in M_{G}$ such that $\sigma_{t}^{\varphi}(x)=v_{t} x v_{-t}$ for $x \in M_{G}$ as in Theorem 7 the $v_{s} \in M_{\varphi}$. But $M_{\varphi}=\mathscr{Z}$ so that all of $M_{G}$ is fixed.

CoRollary 9. With the same setting, if $M_{\varphi}=\{\lambda I\}$ then $M_{G}$ is a factor so that either $M_{G}$ is type III or $M_{G}=\{\lambda I\}$.

Proof. Any central projection in $M_{G}$ is fixed by $t \rightarrow \sigma_{t}^{\varphi}$.

Let us now assume that $\left\{\alpha_{g}: g \in G\right\}$ fixes the states $\varphi$. Then, it follows that $\alpha_{g}$ commutes with $\sigma_{t}^{\varphi}$ [5]. Moreover suppose, without any loss of generality, that $M$ acts on a Hilbert space $\mathfrak{S}$ and $\varphi(x)=$ $\left(x \xi_{\varphi} \mid \xi_{\varphi}\right)$ where $\xi_{\varphi}$ is a cyclic and separating vector for $M$. Then there exists a unitary group $g \rightarrow u_{g}$ such that $\alpha_{g}(x)=u_{g} x u_{-g}$ and $u_{g} \xi_{\varphi}=\xi_{\varphi}$ for all $g \in G$. Let $E_{0}$ denote the projection onto the subspace of $\mathscr{S}$ given by $\left\{\xi: u_{g} \xi=\xi\right.$ for all $\left.g \in G\right\}$. 
Definition. If $E_{0} M E_{0}$ is abelian then the system $\left\{M, \varphi, \alpha_{g}\right\}$ is said to be $G$-abelian [4] and [9].

CoRollary 10. Let $\left\{M, \varphi, \sigma_{t}^{\varphi}, \alpha_{g}\right\}$ be as just described then if $M$ is G-abelian, $M_{G} \cong \mathscr{z}$.

Proof. $E_{0} M E_{0}$ equals, [4], $M_{G} E_{0}$ which is isomorphic to $M_{G}$. The latter being abelian is a fortiori semifinite, hence Theorem 8 applies.

1. The semifiniteness of $M_{G}$ is equivalent to that of $\mathscr{R}\left(M, U_{G}\right)$. For the latter one should see a recent paper of Pedersen and Størmer [11].

What remains is some commentary on the condition $M_{\varphi}=\mathscr{Z}$. Suppose then that $M=\pi_{\varphi}(\mathfrak{Q})^{\prime \prime}$ arises via the GNS procedure [3], from a $C^{*}$-algebra $\mathfrak{A}$ with state $\phi$ and KMS automorphism group $t \rightarrow \sigma_{t}$.

In [5] a condition that $M_{\varphi}=\mathscr{\gamma}$ was given and a class of representation of the Clifford algebra were shown to satisfy ergodicity in time. We offer

THeOREM 11. Suppose $\mathfrak{X}$ is a $C^{*}$-algebra acted upon by a oneparameter automorphism group, $t \rightarrow \sigma_{t}$ which, together with a state $\varphi$, satisfies the KMS boundary condition. If $M=\pi_{\varphi}(\mathfrak{Z})^{\prime \prime}$, then $M_{\varphi}=\mathscr{Z}$ if and only if whenever a state $\psi$ on $\mathfrak{A}$ is invariant under the group $\left\{\sigma_{t}\right\}_{t \in R}$ and $\pi_{\psi}$ is quasi-equivalent to $\pi_{\varphi}$, we have that $\psi$ is a KMS state for $\left\{\sigma_{t}\right\}$.

Proof. Suppose $\pi_{\varphi}$ and $\pi_{\psi}$ are quasi-equivalent and that $\psi$ is invariant. There then exists an isomorphism $\Phi$ taking $\pi_{\psi}(\mathfrak{R})^{\prime \prime}$ onto $\pi_{\varphi}(\mathfrak{R})^{\prime \prime}$ such that $\Phi\left(\pi_{\psi}(x)\right)=\pi_{\varphi}(x), x \in \mathfrak{A}$. Let $\xi_{\psi}, \xi_{\varphi}$ be vectors in $\mathfrak{S}_{\psi}, \mathfrak{S}_{\varphi}$ respectively, such that

$$
\psi(x)=\left(\pi_{\psi}(x) \xi_{\psi} \mid \xi_{\psi}\right) ; \varphi(x)=\left(\pi_{\varphi}(x) \xi_{\varphi} \mid \xi_{\varphi}\right) \quad \text { for } \quad x \in \mathfrak{N} .
$$

Define a normal state $\omega$ on $\pi_{\varphi}(\mathfrak{R})^{\prime \prime}$ by

$$
y \longrightarrow\left(\Phi^{-1}(y) \xi_{\psi} \mid \xi_{\psi}\right) \text {. }
$$

Then, if $x \in \mathfrak{R}$

$$
\omega\left(\pi_{\varphi}(x)\right)=\left(\Phi^{-1}\left(\pi_{\varphi}(x)\right) \xi_{\psi} \mid \xi_{\psi}\right)=\left(\pi_{\psi}(x) \xi_{\psi} \mid \xi_{\psi}\right)=\psi(x) .
$$

Furthermore,

$$
\begin{aligned}
\omega\left(\sigma_{t}\left(\pi_{\varphi}(x)\right)\right) & =\omega\left(\pi_{\varphi}\left(\sigma_{t}(x)\right)\right)=\left(\pi_{\psi}\left(\sigma_{t}(x)\right) \xi_{\psi} \mid \xi_{\psi}\right) \\
& =\psi\left(\sigma_{t}(x)\right)=\psi(x)=\omega\left(\pi_{\varphi}(x)\right) .
\end{aligned}
$$


Thus $\omega$ is an invariant state on $M$. Since $M_{\varphi}=\mathscr{Z}$,

$$
\omega(y)=\left(y h \xi_{\varphi} \mid h \xi_{\omega}\right), y \in M \text { and } h \in \mathscr{\digamma}[16] \text {. }
$$

It is clear then that $\omega$ satisfies the KMS condition on $\pi_{\varphi}(\mathfrak{R})^{\prime \prime}$ and thus $\psi$ does on $\mathfrak{A}$

For the converse let $M=\pi_{\varphi}(\mathfrak{A})^{\prime \prime}$ and consider $h$, nonsingular belonging to $M_{\varphi}$ with $h \geqq 0$. Suppose $\left\|h \xi_{\varphi}\right\|=1$ and let $\xi_{\psi}=h \xi_{\varphi}$. Since $\xi_{\varphi}$ is cyclic and separating $M$ so is $\xi_{\psi}$. Define a state $\psi$ on $\mathfrak{A}$ by

$$
\psi(x)=\left(\pi_{\varphi}(x) \xi_{\psi} \mid \xi_{\psi}\right) .
$$

Clearly the canonical representation $\pi_{\psi}$ of $\mathfrak{A}$, due to $\psi$ is unitarily equivalent to the triple $\left\{\pi_{\varphi}, \xi_{\psi}, \mathfrak{S}_{\varphi}\right\}$ and thus $\pi_{\varphi}$ and $\pi_{\psi}$ are quasiequivalent [3]. Now by assumption $\psi$ is a KMS state on $\mathfrak{A}$ and hence the vector state $\omega_{\xi \psi}$ satisfies the KMS condition with respect to $\sigma_{t}^{\varphi}$ on $M$. It follows, [16], that for $y \in M$

$$
\left(y h \xi_{\varphi} \mid h \xi_{\varphi}\right)=\omega_{\xi_{\psi}}(y)=\left(y k \xi_{\varphi} \mid k \xi_{\varphi}\right) \quad \text { with } \quad k \geqq 0
$$

bounded (since $h \in M_{\varphi}, \psi \leqq \varphi$ ) and belonging to $\mathscr{Z}$. Thus $h=k$ and $M_{\varphi}$ is contained in, and hence equal to $\mathscr{Z}$.

The above theorem was prompted by a question to the author by D. Kastler.

The author would like to thank G. K. Pedersen for bringing reference [10] to his attention, and E. Størmer for conversations about same.

\section{REFERENCES}

1. F. Combes, Poids associés a une algebre Hilbertienne a gauche, Comp. Math., 23, Fasc. 1 (1971), 4-77.

2. A. Connes, Une classification des facteurs de type III, Thesis, to appear.

3. J. Dixmier, Les algebres d'operateurs dans l'espace Hilbertien, Gauthier-Villars, Paris, 1957.

4. S. Doplicher, D. Kastler, and E. Størmer, Invariant states and asymptotic abelianess, J. Functional Analysis, 3 No.3, (1969), 419-434.

5. R. Herman and M. Takesaki, States and automorphism groups of operator algebras, Commun. Math. Phys., 19 (1970), 142-160.

6. R. Herman, Centralizers and an ordering for faithful, normal states, to appear in J. Functional Analysis.

7. R. R. Kallman, Certain topological groups are Type I, Bull. Amer. Math. Soc., 76 (1970), 404-406.

8. I. Kovacs and J. Szücs, Ergodic type theorems in von Neumann algebras, Acta Sci. Math., 27 (1966), 233-246.

9. O. Lanford and D. Ruelle, Integral representations of invariant states, J. Math. Phys., 8 (1967), 1640.

10. R. Nest, Invariant weights of operator algebras satisfying the KMS condition, to appear.

11. G. K. Pedersen and E. Størmer, Automorphisms and equivalence in von Neumann algebras $I I$, to appear. 
12. G. K. Pedersen and M. Takesaki, The Radon-Nikodym theorem for von Neumann algebras, to appear in Acta Math.

13. E. Størmer, Automorphisms and invariant states of operator algebras, Acta Math., 127 (1971), 1-9.

14. - Automorphisms and equivalence in von Neumann algebras, Pacific J. Math., 44 (1973), 371-383.

15. - States and invariant maps of operator algebras, J. Functional Analysis, 5 (1970), 44-65.

16. M. Takesaki, Tomita's theory of modular Hilbert algebras and its applications, Lecture notes in mathematics, Springer-Verlag, V. 128, 1970.

Received October 29, 1973. The preparation of this paper was partially supported by NSF Grant GP-38966.

Pennsylvania State University 



\section{PACIFIC JOURNAL OF MATHEMATICS}

EDITORS

RICHARD ARENS (Managing Editor)

University of California

Los Angeles, California 90024

R. A. Beaumont

University of Washington

Seattle, Washington 98105

J. DugundJI

Department of Mathematics

University of Southern California

Los Angeles, California 90007

D. Gilbarg and J. Milgram

Stanford University

Stanford, California 94305

\section{ASSOCIATE EDITORS}

E. F. BECKENBACH

B. H. NeumanN

F. WOLF

K. YOSHIDA

\section{SUPPORTING INSTITUTIONS}

UNIVERSITY OF BRITISH COLUMBIA CALIFORNIA INSTITUTE OF TECHNOLOGY

UNIVERSITY OF CALIFORNIA

MONTANA STATE UNIVERSITY

UNIVERSITY OF NEVADA

NEW MEXICO STATE UNIVERSITY

OREGON STATE UNIVERSITY

UNIVERSITY OF OREGON

OSAKA UNIVERSITY

\author{
UNIVERSITY OF SOUTHERN CALIFORNIA \\ STANFORD UNIVERSITY \\ UNIVERSITY OF TOKYO \\ UNIVERSITY OF UTAH \\ WASHINGTON STATE UNIVERSITY \\ UNIVERSITY OF WASHINGTON \\ $\stackrel{*}{*} \stackrel{*}{*}{ }^{*}{ }^{*}{ }^{2}$ AMERICAN MATHEMATICAL SOCIETY \\ NAVAL WEAPONS CENTER
}




\section{Pacific Journal of Mathematics}

\section{Vol. 52, No. $1 \quad$ January, 1974}

David R. Adams, On the exceptional sets for spaces of potentials ............ 1

Philip Bacon, Axioms for the Čech cohomology of paracompacta ............ 7

Selwyn Ross Caradus, Perturbation theory for generalized Fredholm operators ..... 11

Kuang-Ho Chen, Phragmén-Lindelöf type theorems for a system of nonhomogeneous equations ............................ 17

Frederick Knowles Dashiell, Jr., Isomorphism problems for the Baire classes .......

M. G. Deshpande and V. K. Deshpande, Rings whose proper homomorphic images are right subdirectly irreducible . . . . . . . . . . . . . . . . . . . . . . . . .

Mary Rodriguez Embry, Self adjoint strictly cyclic operator algebras .............

Paul Erdős, On the distribution of numbers of the form $\sigma(n) / n$ and on some related

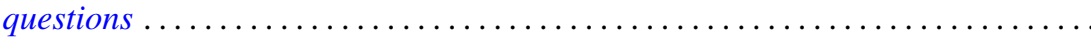

Richard Joseph Fleming and James E. Jamison, Hermitian and adjoint abelian

operators on certain Banach spaces ............................

Stanley P. Gudder and L. Haskins, The center of a poset .................. 85

Richard Howard Herman, Automorphism groups of operator algebras . . . ........

Worthen N. Hunsacker and Somashekhar Amrith Naimpally, Local compactness of families of continuous point-compact relations ....................

Donald Gordon James, On the normal subgroups of integral orthogonal groups ....

Eugene Carlyle Johnsen and Thomas Frederick Storer, Combinatorial structures in

loops. II. Commutative inverse property cyclic neofields of prime-power

order.

Ka-Sing Lau, Extreme operators on Choquet simplexes . . . . . . . . . . . . . . 129

Philip A. Leonard and Kenneth S. Williams, The septic character of 2, 3, 5 and $7 \ldots 143$

Dennis McGavran and Jingyal Pak, On the Nielsen number of a fiber map ........ 149

Stuart Edward Mills, Normed Köthe spaces as intermediate spaces of $L_{1}$ and

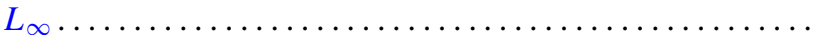

Philip Olin, Free products and elementary equivalence. .

Louis Jackson Ratliff, Jr., Locally quasi-unmixed Noetherian rings and ideals of the principal class.

Seiya Sasao, Homotopy types of spherical fibre spaces over spheres ...

Helga Schirmer, Fixed point sets of polyhedra ...

Kevin James Sharpe, Compatible topologies and continuous irreducible

representations.

Frank Siwiec, On defining a space by a weak base . . . . . . . . . . . . . . . 233

James McLean Sloss, Global reflection for a class of simple closed curves ....... 247

M. V. Subba Rao, On two congruences for primality . .

Raymond D. Terry, Oscillatory properties of a delay differential equation of even

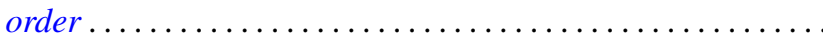

Joseph Dinneen Ward, Chebyshev centers in spaces of continuous functions . .

Robert Breckenridge Warfield, Jr., The uniqueness of elongations of Abelian

groups...

V. M. Warfield, Existence and adjoint theorems for linear stochastic differential

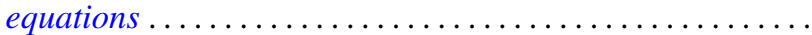

